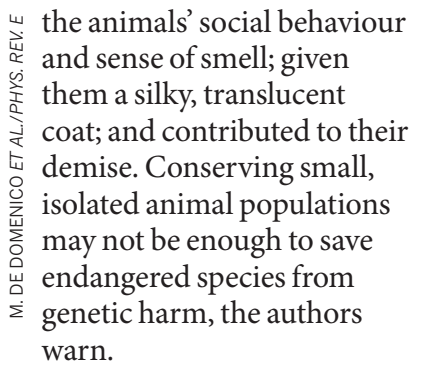

PLoS Genet. 13, e1006601 (2017) TECHNOLOGY

\section{Insects inspire crash-proof drone}

A drone with wasp-like wings can bounce back into shape after crashing.

Drone makers often try to protect their devices by using stiff, bulky frames, but these tend to fail during high-speed impacts. Conversely, wasps have wings with flexible joints that allow the wings to deform during collisions. Stefano Mintchev and his colleagues at the Swiss Federal Institute of Technology in Lausanne created a remote-controlled drone (pictured) with flexible fibreglass arms. During flight, the arms are locked to the central body by magnetic joints to keep them rigid. When subjected to an impact, the joints release, allowing the arms to bend, absorb the shock and snap back into place. Over the course of 50 collisions, the 50-gram prototype sustained damage only twice.

Combining rigid and soft materials into one device could help to improve the resilience of mobile robots, the authors say.

IEEE Robot. Automat. Lett. http://doi.org/b2hm (2017)
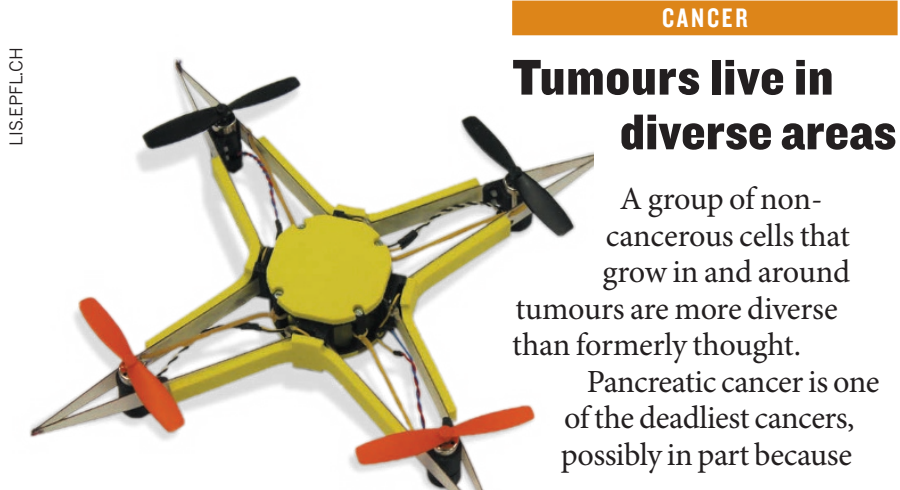

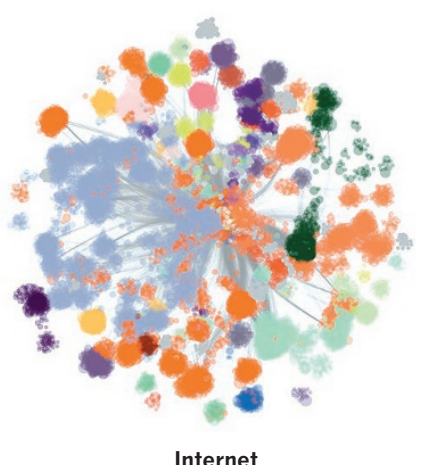

\section{NETWORK THEORY}

\section{How the Darknet defends itself}

The Darknet — the global network that hosts anonymous, and often illicit, online activity - is more resistant to attack than the Internet, thanks to its decentralized structure.

The Darknet routes all data through complex paths using a network of relays to conceal users' identities. Manlio De Domenico and Alex Arenas of Rovira i Virgili University in Tarragona, Spain, analysed the network periodically between late 2013 and early 2015. They report that the Darknet is less centralized than the Internet, routing its traffic more evenly throughout the network, rather than relying on a few core 'hubs' (pictured, with hubs coloured). In simulations of attacks, the researchers found that this structure meant there was less disruption to traffic flow when nodes went down.

However, the Darknet's resilience fell slightly during the study period, suggesting the network is evolving and possibly becoming centralized. Phys. Rev. E 95, 022313 (2017)

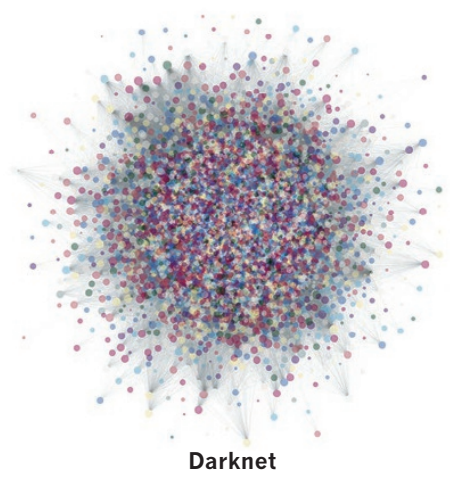

the tumours are surrounded by relatively large amounts of connective tissue called stroma, which promotes their growth. To learn more about the stroma, David Tuveson at Cold Spring Harbor Laboratory in New York and his colleagues grew 3D mouse pancreatic tumours and cultured them with pancreatic cells that develop into connectivetissue cells called fibroblasts. This yielded two subgroups of fibroblasts - one that sits next to and interacts with the cancer cells, and another that is located farther away and produces tumour-boosting inflammatory molecules.

These fibroblasts could have both pro- and anti-tumour effects, which may explain why some therapies that target the stroma fail to curb tumour growth, the authors say. J. Exp. Med. http://doi.org/b2jf (2017)

\section{WILDLIFE MANAGEMENT}

Fear used to manage pests

The number of nuisance geese on a South African golf course can be controlled by exploiting the birds' fear of predators.

Alex Atkins at the Percy FitzPatrick Institute of African Ornithology in Cape Town, South Africa, and his colleagues studied Egyptian geese (Alopochen aegyptiaca) at the Rondebosch Golf Club in suburban Cape Town. They arranged for trained Harris' hawks (Parabuteo unicinctus) to fly at the geese periodically for nine weeks. Hawks killed a few geese, but not nearly enough to account for the observed $73 \%$ decrease in goose numbers. The team noted a $76 \%$ increase in vigilance behaviours by geese after the hawks' introduction, suggesting that fear of the predators had driven most of the birds away.

Goose numbers returned to pre-study levels within two months of the hawks' departure, suggesting that the method requires the continued presence of predators to maintain effect.

J. Wildlife Manage. http://doi.org/ b2h9 (2017)

\section{ARTIFICIAL INTELLIGENCE}

\section{Algorithm masters poker}

An artificial-intelligence (AI) system has beaten professional poker players at a two-player version of the game called Texas hold'em that has no limits on betting.

Poker is hard for machines to master because it has a huge number of possible outcomes and some information - the identity of an opponent's cards — remains unknown. Michael Bowling and his colleagues at the University of Alberta in Edmonton, Canada, created an algorithm, called DeepStack, that learns how likely it is to win in different scenarios by playing against itself in millions of games. During a game, it uses this 'intuition' to explore only a fraction of possible future moves and outcomes, allowing it to form its strategy in real time. The AI system outperformed 10 out of 11 players by a statistically significant amount over the course of the 3,000 games played by each individual.

Such algorithms may one day be used to aid decisionmaking in other areas that lack perfect information, such as defence, say the authors. Science http://doi.org/b2jd (2017) For more on this story, see p.160

\section{$\checkmark$ NATURE.COM}

For the latest research published by Naturevisit:

www.nature.com/latestresearch 